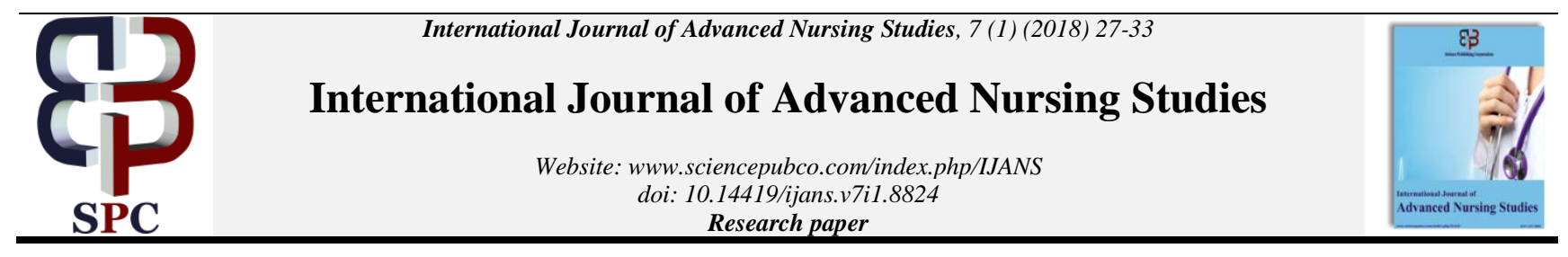

\title{
Nurses' barriers when using research information in clinical decision making
}

\author{
Safaa Mohamed Abdelrahman ${ }^{1}$, Mona Thabet ${ }^{2}$ \\ ${ }^{1}$ Nursing Administration Department, Faculty of Nursing, Minia University, Egypt \\ ${ }^{2}$ Nursing Education Department, Faculty of Nursing, Minia University, Egypt \\ *Corresponding author E-mail: abdelrahman.safaa@yahoo.com
}

\begin{abstract}
Recently, there has been more emphasis on clinical decision making to be a cooperative process, which encompasses shared and parallel decision making with patients and health care teams. For this, nurses' clinical decision-making is a complicated process with a possibility to affect the provided quality of care and. Therefore, affect patient condition progress. Thus, it is the critical point to study the nurses' barriers to research to identify the starting point of how do nurses currently view, and apply research based information in their decision. The aim of the study is to evaluate the nurses' barriers when using research information in clinical decision making. A descriptive correlation research design was utilized. The sample was consisted of of 140 nurse participants at Minia University hospitals. One tool was used as Barriers to using research information in clinical decision making. This study revealed that the nurse participants agree on the research barriers such as lack of time, lack of organization support to use and implement insufficient nurse skills to use research, and complex nature of research. Thus it was concluded that nurses appraise the value of research utilization, but there were many factors hinder and become the barrier to them to implement research.
\end{abstract}

Keywords: Barriers to Research Information; Clinical Decision Making; Evidence Based Decision; Research Utilization.

\section{Introduction}

Today, nurses are increasingly facing complicated challenges when providing health care, which asked them to develop and enhance their skills as problem-solving critical thinking, and decision-making. These skills are keys attributes for nurses, in which help them to in the delivery of health care and improve nurses' skillfulness (Hoffman\& Elwin 2004).Also, Furthermore, the quickly expansion in nursing practice has a great confirmation on nurses' autonomy in health care delivery and making them to have more responsibility in deciding the consequences of their interventions for patient care (Ludin 2017).

Moreover, there has been more remarkable increasing of nursing knowledge in the last decades, as well the nurses' number with high academic certifications is continuously progressed. So, it is crucial for the extension of nursing knowledge to discover new methods that will ensure research information and evidence based knowledge reach the practitioners' nurse, for the implementation and evaluation (Kajermo et al. 1998). Furthermore, as the needs of patients' health care become more complicated and sophisticated, nurses must be able to have the high degree of competence, proficiency and self-confidence when providing care as well when make clinical decisions (Woda et al. 2017).

Decision making is an expansive issue which defines as a process of making a choice between variety of alternative and options to have a significant action. So, health professionals have to make clinical decisions, which are more sophisticated process, and demanding more skills and efficiency from individuals than making known choices between fixed alternatives. Health care professionals are asked to take decisions with multiple concentrations on patients' needs, in the dynamic process, using a variety of knowledge base, which is based on evidence literature, and with multiple individuals and information involved (Higgs et al. 2008). In addition, clinical decisions are described by vague and ambiguous conditions in which all the information needed to make the suitable decisions is not clear, not found, or not known. In this context, the clinical decision making has rarely single decisions that cannot be made from a fixed choice. Additionally, the decisions and alternatives have to influence each other. Recently, there has been more emphasis has been positioned on clinical decision making to be cooperative process, that encompasses shared and parallel decision making with patients and health care professionals teams (Edwards et al. 2004).

For nurses, clinical decision-making are the decisions that nurses make for providing patient care (Choi

2001).Forthis,hnurses'sclinicalidecision-

makingkisgaicomplicatedaprocesscwithwatpossibilityltoyaffectfthe providediqualitylofycare-

cand,atherefore,oaffectfpatienticonditiontion progreEffectivetclinicalidecision-makingkisgpromotedooverotimetasenursesrgaingthe necessarysskills, lthinkingkprocesses,sknowledge, dand clinicaliexperiencee (Tannern2006).06).

Clinical decision-making had many studies that explore for nurses multiple processes and diverse theoretical point of view. These studies suggest that clinical decision making is including three sequential stages: observation of patient condition, assessment of observations, and taking action to achieve desired outcomes. Thus, nurses are the first one of the health care professionals who monitors the deterioration in physical conditions of patients well, they are in a position to use their knowledge and experience to meet the needs' patients (Lauri\&Salantera 2002 and Choi\& Kim 2015).

There are two theories of decision-making that have been used in the nursing as analytical- systematic and intuitive-interpretive

Copyright () 2018 Safaa Mohamed Abdelrahman, Mona Thabet. This is an open access article distributed under the Creative Commons Attribution License, which permits unrestricted use, distribution, and reproduction in any medium, provided the original work is properly cited. 
decision-making theories (Choi\& Kim 2015).Theanalytical- systematic theory depicts the cognitive processing in clinical decision-making and presumes, that persons can shift through a number of stages in the decision-making process. The analytical- systematic theory supposes that nurses use a hypothetical-deductive approach in clinical decision-making. On the other side, intuitiveinterpretive decision-making theories center around the relationship between surmise, intuition and nursing knowledge (Lauri et al. 2001 and Banning 2008).These theories suggested that the process of making decisions is grasped on a continuum from analysis to intuition, as it is based on analytical-systematic and intuitive-interpretive theories (Choi 2001).

Moreover, there are three models of clinical decision-making that explain and clarify the decision-making process nurses can use to reach choice. Three major models that found throughout the literature are the humanistic-intuitive model, the informationprocessing model, and the cognitive continuum theory. The humanistic-intuitive approach confirms personal, sense, and contextual elements in decision-making. The basic focus on this model is to explain the changes in making decision between novice and expert nurses. In the information-processing model, decisions are accomplished in an orderly and analytical pattern. They go ahead with a series of steps that portray cue acquisition, hypothesis generation, cue interpretation, and hypothesis evaluation. The cognitive continuum theory servers to bear down the totally differences between systematic-analytical and intuitive approaches by feigned that both are required to achieve most favorable decisions (Cader et al. 2005and Muntean 2012).

One of the most critical and vital components of nursing practice is clinical decision making is. As, when nurses have the ability to make professional clinical decisions, results in dismiss of medication errors and patient mortality, as well as can be increasing nurses' ability to recognize a deteriorating patient status and increased patient safety (Lauri\&Salantera 2002 and Dickson\& Flynn 2015). Clinical decision-making have an important and crucial role in the quality of care that provided to patients by nurses. Sequentially, poor decision-making can lead to inverse effect as well have negative outcomes for patients. It is rated that up to $65 \%$ of inverse effect could be limited and prohibited when nurses made better decisions (Hodgetts et al. 2002and Brennan et al. 2004).

Historically, patient' care was influenced by the experiences, knowledge, thinking and view of those who providing treatment for a patient (Kania-Lachance et al. 2006). Evidence-based practice can help health-care professional to change from a traditional emphasis on authoritative views to the confidence data, taken away from prior research and studies (Jette et al. 2003 and Majid et al. 2011). In addition, when nursing practices are based on evidence, it can improve patient care, as compared to traditional practices. Moreover, as nurses are asked more and more to be involved in clinical decision making, it is becoming important issue for them to apply and use the best evidence to make functional, efficient, operative, and justifiable decisions (Mantzoukas 2007 and Majid et al. 2011).

There are various issues that support nurses in clinical decision making, nursing care and their capacity to build knowledge and search for information. Firstly, patient status knowledge provides direction for knowing the condition of patient. Deficit's information will limit the nurses' ability to build correctly decisions Secondly, nurses may have sufficient information but, because of the poor process of thinking, they have difficulty applying the research information and build decision-making. Thirdly, experience of nurses will affect their abilities to take decisions (Del Bueno 2005and Gillespie 2010).

Therefore, nurses must have evidence-based decision making, which should integrate the information and knowledge arising from one's clinical experiences, preferences of a patient, and research evidence within the context of available data. In making evidence-based decisions, searching for evidences mustn't be taken at face value and abides by uncritically, but there should be appropriate weight in a decision relying on its internal and external validity. Combining research evidences when making decision include forming a basic clinical question, inspecting for the most convenient evidence to meet patient's need, critically appreciates the available evidence, integrating the evidence into action, and assessing the effects of decisions and actions taken (Thompson 2004).

Overwhelmingly, time is one of the most important barriers to research utilization in clinical decision making when provide nursing practice, as well lack of support, motivation and independence for the nurses in the hospital organization is perceived to block initiatives for nursing research application. Furthermore, the presentation of research can be perceived as a barrier due to its difficulty to read, sophisticated statistics, and no clear significance showed in research to nursing practice. Furthermore, the absence or lack of nurses skills in research utilization, nurses inexperience to narrow the search data without lost significant information, and doubt of nurses when to stop searching for information are other barriers in research information (Kajermo et al. 1998 and Haynes et al. 2005).

\section{Significance of study}

Clinical decision making that is based on evidence-based considered as a prescriptive approach to making choices, is broadly advocated as an ideal for clinical practice, and is focused upon the concept of how theory can be utilized to improve the actual world of making decision. However, before developing a plan to attain the ideal clinical decision making that is based on evidence and research information, it is important to identify the starting point. As how do nurses currently view, utilize, and apply research based information in decision making? Are there barriers that hindering nurses for using research in clinical decision making? Surprisingly in Upper Egypt, there are still little numbers of researches that have been done on this search the nurses use of evidence and barriers that can hinder them using research information.

\section{Aim}

- To evaluate the nurses' barriers when using research information in clinical decision making.

\section{Research questions}

- What are nurse's barriers when using research information in clinical decision making?

- Is there relationship between the barriers of using research information in clinical decision making and nurses' sociodemographic characteristics?

\section{Material and methods}

\subsection{Design}

Descriptive design was utilized in the current study.

\subsection{Setting}

This study was conducted at Minia University Hospitals, Miniacity. It was including three hospitals as: Minia University Hospital, Pediatric and obstetric University Hospital, and Urology University Hospital, including all departments.

\subsection{Subjects}

A convenience sample of participated nurses in three hospitals, the total number was 140 nurses divided into (staff nurse $=115$, head nurse $=18$, supervisors $=3$, and managers $=4$ ). 


\subsection{Study tool}

One study tool was used as "Barriers to using research information in clinical decision making"it was developed by (McCaughan et al. 2002) to measure nurses' opinions about the barriers when using research information in clinical decision making. It was including 60 items, divided by the researchers into 5 dimensions as: Nature of research as the problem (12 items); Credibility and applicability of research (14 items); Beliefs and value of nurses about research (9 items); Nurseskills and experiences with research (9 items); and Culture and organization barriers (14 items). Also, the cover about socio-demographic characteristics was attached to tool including data as age, gender, marital status, years of experience, level of education, and current position.

\subsection{Scoring system}

The response to each item in the questionnaire was assessed by using three points Likert scale as $(3=$ agree, $2=$ neutral, and $1=$ disagree). The scoring system was ranging 60 to 180 , as (60-100) low agreement of research information barriersin clinical decision making, (101-140) moderate agreement of research information barriers in clinical decision making, and (141-180) high agreement of research information barriers in clinical decision making, the higher score, the higher agreement of research information barriers in clinical decision making.

\subsection{Pilot study}

A pilot study was conducted on $10 \%$ of participants who was equal approximately 15 nurses to ascertain the clarity, comprehensiveness and applicability of the tool as well as to verify that items in the tool had its applicability, validity and clarity. The pilot study applied was not included in the total study subjects.

\subsection{Data collection}

Data collected from 1st July to 31th August 2017.

1) Permission to conduct the study was obtained from all responsible authorities at Minia University Hospitals and Ministry of Health Hospitals after explaining the purpose of the study.

2) The validity of the tool was performed by experts in the related fields. Content validity of the tool was obtained by revision of 3 expert's field of Nursing Administration 2 from Ain shams University and 1 from Cairo University.

3) Reliability of the tool by Cronbach's alpha was 0.95 .

\subsection{Ethical considerations}

The necessary steps were taken to ensure that the rights of all subjects were recognized and protected throughout the study.
Ethical approval was granted from the research ethics Committee, Faculty of Nursing, Minia University.

The participants were informed that their participation in the study was completely voluntary and there will be no harm if they not participate in the study.

Informed consent was obtained from participating nurses after explaining the nature and benefits of the study.

Each assessment sheet was coded and nurses names was not appear on the sheets for the purpose of anonymity and confidentiality.

\subsection{Statistical analysis}

The statistical tests for data analysis as mean, standard deviation, Chi square, and Spearman's rank correlation Coefficient; Pearson Product Moment Correlations were used to analyses the data for this research. All data of the study were fed into an IBMCompatible personal computer using SPSS 20 version.

\section{Results}

Table (I) and figures (I and II) show the distribution of participants' socio-demographic characteristics, as the gender of the participated nurses $(75 \%)$ were female and the highest percent age (69.3\%) of them were 25+ years old. Regarding the participated nurses' educational level and years of experiences were $48.6 \%$ and $46.4 \%$ of them had diploma nursing degree and 1-5 years of experiences respectively. The highest percentage of the current position were staff nurses $(82.1 \%)$ and were $(59.3 \%)$ of them married. Table (II) shows the mean scores of total dimensions of research information barriers in clinical decision making. The dimensions were nature of research as problem, credibility and applicability or research, beliefs and value or research, nurse' skills and experiences with research, and culture and organization barriers, and mean scores were $(26.36,30.37,19.78,20.66$, and 31.29 respectively) with highly statistically significant $\mathrm{P}$ value $=0.000$ for all dimensions.

Figure (III) showed the total score of research information barriers in using clinical decision making, the highest percentage $(77.1 \%)$ of participated nurses agree on research information barriers when using clinical decision making and $(8.6 \%)$ of them had low agreement about barriers in clinical decision making.

Table IV showed that the highest mean scores were high level of barriers in clinical decision with socio-demographic characteristics, as regards to age, gender, years of experience, level of education and current position $(1.67 \pm .47,1.22 \pm .42,1.83 \pm .83,1.87 \pm .88$ and $1.43 \pm .74)$ respectively. There were highly statistically significant differences between levels of research information barriers in clinical decision and socio-demographic characteristics.

Table 1: Distribution of Socio-Demographic Characteristics of the Nurse Participants

\begin{tabular}{|c|c|c|}
\hline \multirow{2}{*}{ Socio-demographic Characteristics } & \multicolumn{2}{|c|}{ Participated Nurses $\mathrm{N}=140$} \\
\hline & $\mathrm{N}$ & $\%$ \\
\hline \multicolumn{3}{|l|}{ Age } \\
\hline$<25$ & 43 & 30.7 \\
\hline $25+$ & 97 & 69.3 \\
\hline Mean \pm SD & \multicolumn{2}{|c|}{$30.07 \pm 6.74$} \\
\hline \multicolumn{3}{|l|}{ Marital status } \\
\hline Single & 49 & 35.0 \\
\hline Married & 83 & 59.3 \\
\hline Divorced & 1 & .7 \\
\hline \multicolumn{3}{|l|}{ Years of experiences: } \\
\hline $1-5$ years & 65 & 46.4 \\
\hline $5-10$ years & 42 & 30.0 \\
\hline More than 10 years & 33 & 29.6 \\
\hline \multicolumn{3}{|l|}{ Levels of education: } \\
\hline Diploma & 68 & 48.6 \\
\hline Technical nurse & 35 & 25.0 \\
\hline
\end{tabular}




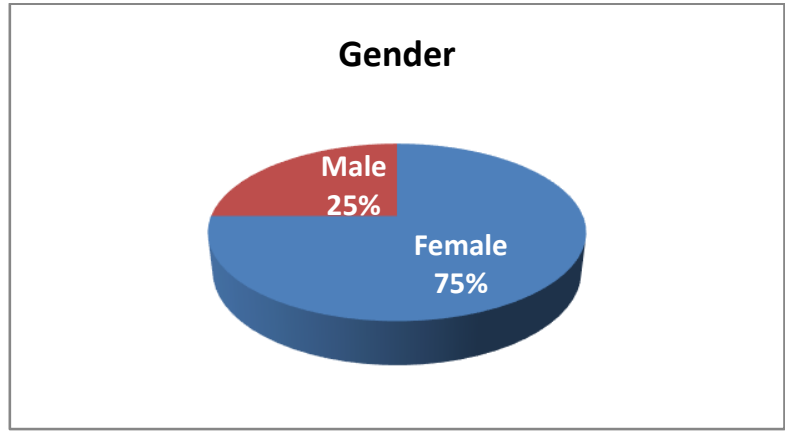

Fig. 1: The Gender of the Nurse Participants $(\mathrm{N}=140)$.

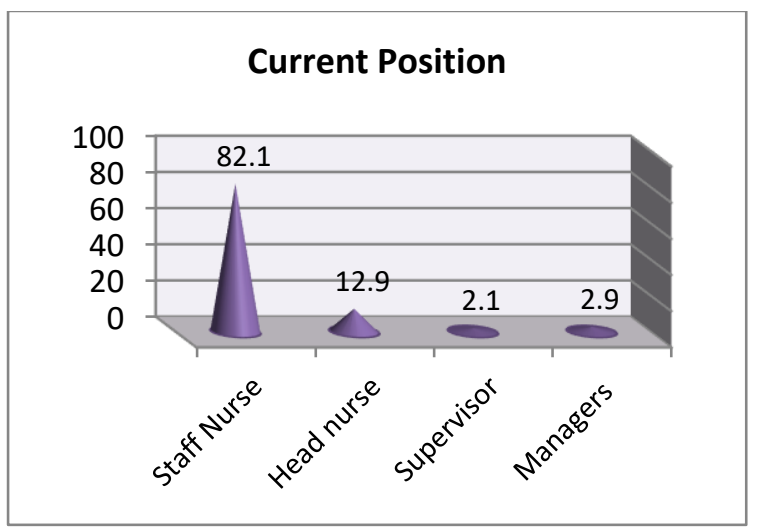

Fig. 2: The Current Position of the Nurse Participants $(\mathrm{N}=140)$.

Table 2: Mean Scores of Total Dimensions of Clinical Decision Making Barriers Fornurse Participants $(\mathrm{N}=140)$

\begin{tabular}{|c|c|c|c|c|c|c|}
\hline \multirow{2}{*}{$\begin{array}{l}\text { Dimensions of Research Infor- } \\
\text { mation Barriers }\end{array}$} & \multicolumn{4}{|c|}{ Mean score + SD } & \multicolumn{2}{|c|}{$95 \%$ confidence of the interval } \\
\hline & Mean & SD & T-test & $\mathrm{P}$ & Lower & Upper \\
\hline Nature of research as problem & 26.36 & 3.89 & 80.13 & 0.000 & 25.71 & 27.01 \\
\hline $\begin{array}{l}\text { Credibility and applicability of } \\
\text { research }\end{array}$ & 30.37 & 5.50 & 65.26 & 0.000 & 29.45 & 31.29 \\
\hline Beliefs and value of research & 19.78 & 3.16 & 73.88 & 0.000 & 19.25 & 20.31 \\
\hline $\begin{array}{l}\text { Nurse skills and experiences with } \\
\text { research }\end{array}$ & 20.66 & 3.32 & 73.61 & 0.000 & 20.10 & 21.21 \\
\hline Culture and organization barriers & 31.29 & 4.63 & 79.87 & 0.000 & 30.51 & 32.06 \\
\hline
\end{tabular}

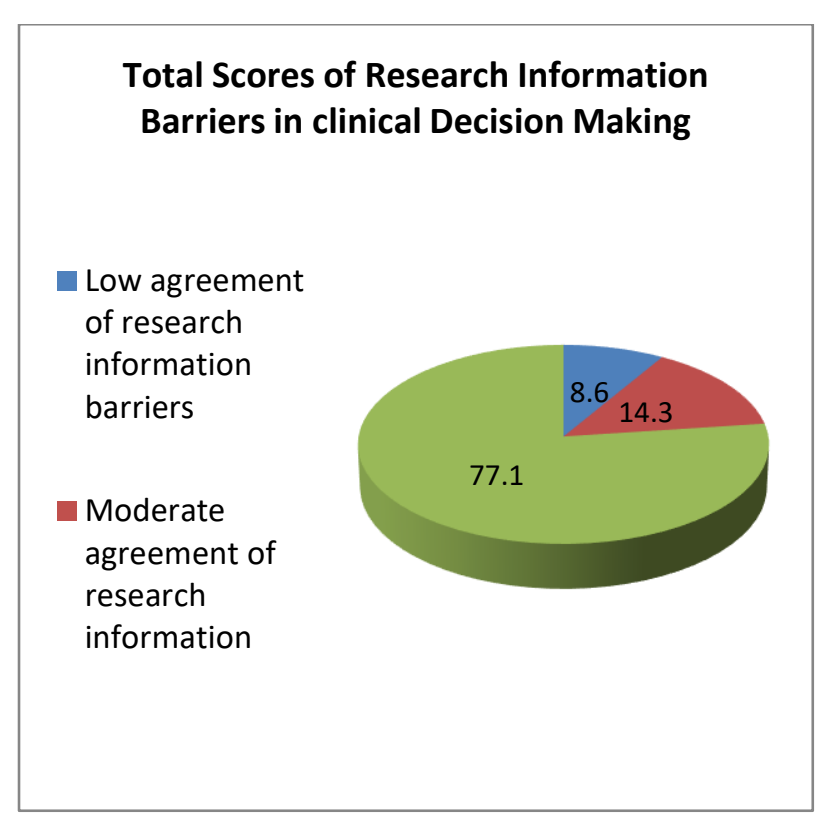

Fig. 3: Total Score of Research Information Barriers in Using Clinical Decision Making among Nurse Participants (N=140).

Table V showed that there was statistically significant difference between gender and culture and organizational barriers in favor to female $(31.9 \pm 4.4)$, with $\mathrm{p}$ value $=0.004$. Regarding the level of education, there was statistically significant difference between level of education and four dimensions as; nature of research as problem (mean=27.6 \pm 3.3 , with $\mathrm{P}=0.002)$, credibility and applica- bility or research $($ mean $=32.7 \pm 4.45$, with $\mathrm{P}=0.002)$, beliefs and value or research (mean $=20.7 \pm 2.6$, with $P=0.01)$, nurse' skills and experiences with research $($ mean $=21.9 \pm 2.3$, with $\mathrm{P}=0.02)$ in favor to BSCs degree. Regarding the current position, there was statistically significant difference between current position and four dimensions as; nature of research as problem (mean $=29 \pm 1.7$, with 
$\mathrm{P}=0.02)$ in favor to head nurses, while credibility and applicability or research $($ mean $=33.7 \pm 5.5$, with $\mathrm{P}=0.01)$, nurse' skills and experiences with research (mean $=23 \pm 1.8$, with $\mathrm{P}=0.006)$, and culture and organizational barriers (mean $=32.4 \pm 4.1$, with $\mathrm{P}=$ 0.001 ) were in favor to supervisors.

Table 4: Relations between Barriers Levelin Clinical Decision Making and Their Characteristics of Nurse Participants (N=140)

\begin{tabular}{|c|c|c|c|c|}
\hline \multirow{3}{*}{ Socio-demographic characteristics } & \multicolumn{4}{|c|}{ Level of Barriers in Research Information } \\
\hline & Low & High & $\mathrm{F}$ & $P$ value \\
\hline & Mean \pm SD & Mean \pm SD & & \\
\hline Age & $1.75 \pm .45$ & $1.67 \pm 47$ & 2.06 & 0.002 \\
\hline Gender & $1.5 \pm .52$ & $1.22 \pm .42$ & 2.03 & 0.002 \\
\hline Marital status & $1.66 \pm .49$ & $1.69 \pm .63$ & .997 & 0.92 \\
\hline Years of experience & $1.42 \pm .67$ & $1.83 \pm .83$ & 1.81 & 0.008 \\
\hline Levels of education & $1.50 \pm .52$ & $1.87 \pm .88$ & 2.18 & 0.001 \\
\hline Current position & $1.50 \pm 1.16$ & $1.43 \pm .74$ & 2.02 & 0.002 \\
\hline
\end{tabular}

$* *(\mathrm{P} \leq 0.001)$

Table 5: Relation between Socio-Demographic Characteristics of Nurse Participants and the Research Berries of Information Used in Clinical Decision Making

\begin{tabular}{|c|c|c|c|c|c|}
\hline \multirow{3}{*}{$\begin{array}{l}\text { Socio-demographic char- } \\
\text { acteristics }\end{array}$} & \multicolumn{5}{|c|}{ Dimensions of research barriers in C.D.M } \\
\hline & $\begin{array}{l}\text { Nature of research as } \\
\text { problem }\end{array}$ & $\begin{array}{l}\text { Credibility } \\
\text { \&applicability of re- } \\
\text { search }\end{array}$ & $\begin{array}{l}\text { Beliefs and value about } \\
\text { research }\end{array}$ & $\begin{array}{l}\text { Nurse Skills \& experi- } \\
\text { ences }\end{array}$ & $\begin{array}{l}\text { Culture \& organi- } \\
\text { zation barriers }\end{array}$ \\
\hline & Mean \pm SD & Mean \pm SD & Mean \pm SD & Mean \pm SD & Mean \pm SD \\
\hline \multicolumn{6}{|l|}{ Age } \\
\hline$<25$ & $26.2 \pm 4.3$ & $29.9 \pm 5.3$ & $19.9 \pm 3.4$ & $20.3 \pm 3.3$ & $32.4 \pm 4.6$ \\
\hline $25+$ & $26.5 \pm 3.8$ & $30.5 \pm 5.6$ & $19.8 \pm 3.1$ & $20.8 \pm 3.3$ & $30.7 \pm 4.6$ \\
\hline F-test (P) & $0.20(0.65)$ & $0.44(0.50)$ & $0.05(0.80)$ & $0.64(0.42)$ & $3.73(0.05)$ \\
\hline \multicolumn{6}{|l|}{ Gender } \\
\hline Females & $26.65 \pm 3.85$ & $30.75 \pm 5.70$ & $20.1 \pm 3.3$ & $20.8 \pm 3.3$ & $31.9 \pm 4.4$ \\
\hline Males & $25.48 \pm 3.92$ & $29.22 \pm 4.74$ & $18.97 \pm 2.7$ & $20.2 \pm 3.5$ & $29.4 \pm 4.8$ \\
\hline F-test $(\mathrm{P})$ & $2.4(0.123)$ & $2.02(0.157)$ & $3.1(0.08)$ & $1.15(0.285)$ & $8.44(0.004)$ \\
\hline \multicolumn{6}{|l|}{ Years of experiences } \\
\hline $1-5$ years & $25.9 \pm 4.2$ & $29.4 \pm 5.3$ & $19.3 \pm 3.4$ & $20.5 \pm 3.4$ & $31.4 \pm 4.6$ \\
\hline $5-10$ years & $26.4 \pm 3.8$ & $32.1 \pm 6.3$ & $19.9 \pm 3.3$ & $20.9 \pm 3.4$ & $30.7 \pm 4.6$ \\
\hline 10 years and more & $27.2 \pm 3.4$ & $31.5 \pm 4.6$ & $20.6 \pm 2.2$ & $20.8 \pm 3.2$ & $31.9 \pm 4.7$ \\
\hline F-test $(\mathrm{P})$ & $1.17(0.31)$ & $2.08(0.12)$ & $2.08(0.12)$ & $0.138(0.87)$ & $0.69(0.50)$ \\
\hline \multicolumn{6}{|l|}{ Levels of education } \\
\hline Diploma & $26.2 \pm 4.02$ & $30.1 \pm 5.99$ & $19.8 \pm 3.5$ & $20.2 \pm 3.5$ & $31.2 \pm 5.1$ \\
\hline Technical & $25.2 \pm 3.9$ & $28.4 \pm 4.66$ & $18.6 \pm 2.7$ & $20.2 \pm 3.4$ & $30.7 \pm 4.6$ \\
\hline BSCs & $27.6 \pm 3.3$ & $32.7 \pm 4.45$ & $20.7 \pm 2.6$ & $21.9 \pm 2.3$ & $32.1 \pm 3.7$ \\
\hline F-test (P) & $3.77(0.02)$ & $6.28(0.002)$ & $4.23(0.01)$ & $3.73(0.02)$ & $0.79(0.45)$ \\
\hline \multicolumn{6}{|l|}{ Current position } \\
\hline Staff Nurses & $26.23 \pm 3.8$ & $29.9 \pm 5.5$ & $19.7 \pm 3.2$ & $20.4 \pm 3.4$ & $31.5 \pm 4.4$ \\
\hline Head nurses & $29 \pm 1.7$ & $32 \pm 2$ & $19.7 \pm 1.5$ & $21.3 \pm 1.5$ & $29.3 \pm 4.7$ \\
\hline Supervisors & $27.7 \pm 4.1$ & $33.7 \pm 5.5$ & $21 \pm 2.9$ & $23 \pm 1.8$ & $32.4 \pm 4.1$ \\
\hline Managers & $21.7 \pm .95$ & $25.8 \pm 1.5$ & $17.5 \pm 1.9$ & $18.3 \pm 3.6$ & $22.5 \pm 4.1$ \\
\hline F-test (P) & $3.3(0.02)$ & $3.56(0.01)$ & $1.64(0.18)$ & $4.31(0.006)$ & $5.94(0.001)$ \\
\hline
\end{tabular}

\section{Discussion}

The concept of using evidence when searching for information is the basis for professional decision-making in the recent days, and become a critical part of nursing's professionalization. Despite primary acceptance of nurses about the value of research information in clinical decision making, it is still known that nurses for many factors and obstacles do not seem to integrate the results getting from research information into the clinical decisions they made. As well the literatures about research information barriers studies still need more studies and investigation (McCaughan et al. 2002).

The current study revealed that the dimensions of research information barriers, which were nature of research as problem, credibility and applicability of research, beliefs and value ofnurse about research, nurse' skills and experiences with research, and culture and organization barriers, had highly been statistically significant, as well the highest percentage (77.1\%) of participated nurses agree on research information barriers when using clinical decision making. This was due to many factors in hospital and nurses beliefs that hinder research information as lack of time to read and search for information, lack of libraries facilities in hospital, insufficient skills in research and computer skills, and difficulties of researches credibility.

This was in line with (Hutchinson and Johnston 2004) who found in their study that the majority of respondents in their study rated barriers items as moderate or great barriers, the most barriers to research information utilization included time bonds, no or weak awareness of available literature in research, having no authority to change their practice, inappropriate skills to judge and criticize the appraisal and insufficient support to implement their research findings.

For the first dimension nature of research as the problem dimensions, the participants reported the most barriers researching information were, "research information is too academic", "statistics put me off research papers or other kinds or research information", "Journals are difficult to read and there are better ways of presenting information than that". This was in congruence with (McCaughan et al. 2002). They found during their interview with nurse participants that statistical of research findings was negative issue to read and apply researches findings. Furthermore, they found that occasionally the language was a barrier to use research information, as well as the overwhelming of research information in both presentation style and its volume. Moreover (Hall 2011), have been reported that staff nurses agree on the highest barriers to the utilization of research was the poor understanding of how to read and use research findings.

For second dimension credibility and applicability of research dimension, the most participant barriers were "I read a paper if it is written by a nurse rather than a Professor as they are more relevant", "I find the research published in medical journals more use than that in the Nursing Times and the other nursing journals", "Research is only limited to a small bit of nursing practice - 
mainly procedures, and we do much more than that", and "Policies and procedures are unwieldy, and once you work here a while you get to know them anyway". This was similar to (Retsas 2000) revealed that the accessibility of research findings filled the majority number of barrier items. Similar (McCaughan et al. 2002), they found that nurse participants in their study reported that research findings had no sufficient guidance and information for their clinical practice and clinical decision making. Moreover, it was found by (Chen et al. (2013) that the top barrier to use research information among Taiwanese nurses was the characteristic of having no trust and sureness to appreciate and use research evidence.

For third dimension the beliefs and value of nurse about research dimension, the greatest barriers to nurses were; "the decisions I make are really complicated and research is often too simple to be of any use", "being able to use research doesn't make you a better nurse", "implementing research is not often led by nurses who are good practitioners", "I think that knowledge gained through experience is more useful than research". Regarding this, (Thompson 2003) mentioned in his study that most of the nurse participants have depended on their experiences and experimental knowledge as the origin of their clinical decisions that they make during their daily practice.Also, (Hutchinson and Johnston 2004), found great barriers due to nurses beliefs as "nurses see little benefit for self", and "nurse does not feel capable of evaluating the quality of the research" which consequentially affect nurses use and apply research findings.

Moreover, about the fourth dimension nurse' skills and experiences with the research dimension, the most barriers form nurse participants views were; "Whenever I have tried to use research in the past, it costs me too much money, time or commitment - like getting papers from the library or whatever", "It's better to have somebody else pass on the research-based messages for practice rather than try and do it yourself"', "I don't know enough about what is available to help me implement research at the hospital", and "I make better decisions without using research. Practice is better dealt with in the here and now". Similarly, (Retsas 2000) illustrated in his study that a great number of nurse participants feeling that they haven't the capability to evaluate the research and its findings, as well the research finding cannot be popularized to their clinical setting. Furthermore, (Sarabia-Cobo et al. 2015) mentioned that the highest mean scores were that nurses have no enough time to their job to implement new ideas and researchers, followed by little knowledge about nursing research.

For the fifth dimension about culture and organization barriers, techniques berries percent barriers, technique's views were, "we don't have the facilities use research in the ward itself. It be better computers and CD-ROMS on the wards rather than somewhere else", "there are people and resources available help implement research but youcan never hold of them when need them", is no real commitment to the part of management to help us get to grips with using research,in our work", "All the facilities, which would help me use research based information are off-site and so difficult to access, and The library is not geared up for nursing research, in.no enough time to their job to implement new ideas and researchers, followed by little knowledge about nursing research.

Thus, it is very important barrier or facilitator to research utilization is the organization and its culture, policy, philosophy and beliefs or its staff. McCaughan et al. (2002) agreed that facilities to help nurses implement their research were not ready on their ward. Also, a major barrier was time as a primary block on their ability in which, they stated that nurses could not use written information of researchers because it is time-consuming to be useful for the clinical practice, as well the inaccessibility of research information or different forms of research basis such as posters, files, protocols, journal articles, books, etc.

Moreover (Retsas 2000), reported significant barriers nursing participants were to their organization, as there is a need from organization to support nurses in implementation and utilization of research information. According to (Mehrdad et al. 2008) the major barriers to use research information were that the lack of nurs- es time to examine researches; organization facilities are insufficient for implementation; and nurses feel they have no enough authority to change clinical care practice for a patient after implementing research.

Furthermore, (Kajermo et al. 2008) have found a great dissatisfaction to the support of supervisors and administrators for the participation and development of researchers, as well as; there were no clear or unrealistic workplace objectives to implement research utilization and evidence in their clinical decision making. Also, (Chau et al. 2008) revealed that all respondents agreed on the highest barriers to research utilization were due to organization factors, which can encompass the inadequate resources, lack of time, no of authority to affect the practice, and lack of participation from physicians to share in clinical decision making.

Furthermore, (Tan et al. 2012) determined that the most important barrier perceived by the nurses was lack of time to do research and use finding from research. And (Sarabia-Cobo et al. 2015) mentioned that a major barrier to nurses in research utilization was "Doctors do not cooperate in the implementation." In addition (Cidoncha-Moreno and Alegría-Fernandez 2017) stated that barriers for nurses were the external factor which related to their organization, as it hinder the implementation of research findings into clinical practice.

Current study showed that there was statistically significant difference between gender and culture and organizational barriers dimension in favor to female, this was due to the majority of participated nurses were females. Regarding the current position, there was statistically significant difference between current position and four dimensions of research information barriers as; nature of research as the problem in favor to head nurses, while credibility and applicability or research, nurse' skills and experiences with research, and culture and organizational barriers were in favor to supervisors. This result indicates that the nurse participant agrees with the importance of research utilization in clinical decision making and had a belief about its value, but there are factors hindering them to use research such as lack of their organization environment support, the research nature, and the research credibility.

\section{Conclusions}

The current study showed that clinical decision making is very crucial aspect in clinical practice for nurses, however there are many factors that affect and become barriers to research utilization among nurses.These factors are multidimensional, and indicate that the responsibility of optimizing them should be shared between researchers, managers, and clinicians. Comprehensively, nurses have been understood the worth of research and proper implementation research findings information; but, they stated significant barriers for them as the need for managers support to have the authority to change patient care, lack of time to appraise and implement research, complexity of research statistics and information, nurse experiences from practice is more useful than research, and no real commitment from management to help nurses to use research in their work.

\section{Recommendation}

- The need for professional development programs that enhance nurses' awareness of the importance of research utilization in clinical decision-making and their clinical practice.

- Further researches with larger numbers to test the generalization from the study results.

- Developing guidelines and procedures for hospitals nurse managers' in order to help their staff nurses implement research utilization in clinical decision-making and their clinical practice. 


\section{Conflict of interests}

The authors have no conflict of interests to declare.

\section{Acknowledgement}

The authors gratefully acknowledge the participants who participate in the pilot study.

\section{References}

[1] Banning M. A review of clinical decision making: Models and current research. Journal of Clinical Nursing. 2008; 17(2): 187-95.

[2] Brennan TA, Leape LL, Laird NM, Hebert L, Localio AR, Lawthers AG, Hiatt $\mathrm{H}$. Incidence of adverse events and negligence in hospitalized patients: Results of the Harvard Medical Practice Study I.Quality\& Safety in Health Care. 2004; 13: 145- 51. https://doi.org/10.1136/qshc.2002.003822.

[3] Cader R, Campbell S, Watson D. Cognitive Continuum Theory in nursing decision-making. Journal of Advanced Nursing. 2005; 49:397-405. https://doi.org/10.1111/i.1365-2648.2004.03303.x.

[4] Chau J, Lopez V, Thompson DR. A Survey of Hong Kong Nurses' Perceptions of Barriers to and Facilitators of Research Utilization. Research in Nursing \& Health. 2008; 31(16):640-9. https://doi.org/10.1002/nur.20289.

[5] Chen SH, Shao JH, Hsiao YC, Lee HC. Barriers to Research Utilization by Registered Nurses in Taiwan. Research in Nursing \& Health. 2013; 36(2):191-202. https://doi.org/10.1002/nur.21521.

[6] Choi HJ. Public health nurses' decision making models and their knowledge structure. Journal of Korean Academy of Nursing. 2001; 31, 328-39. https://doi.org/10.4040/jkan.2001.31.2.328.

[7] Choi M, Kim J. Relationships between Clinical Decision-Making Patterns and Self-Efficacy and Nursing Professionalism in Korean Pediatric Nurses. Journal of Pediatric Nursing (2015) 30: e81-e8. https://doi.org/10.1016/j.pedn.2015.07.001.

[8] Cidoncha-Moreno MA, Alegría-Fernandez BR. Barriers to the implementation of research perceived by nurses from Osakidetza. EnfermClin. $2017 \quad$ Sept; $27(5): 286-93$ https://doi.org/10.1016/j.enfcli.2017.03.015.

[9] Del Bueno D. A crisis in critical thinking. Nursing Education Perspectives. 2005; 26 (5): 278-82.

[10] Dickson GL, Flynn L. Nurses' clinical reasoning: processes and practices of medication safety. Qual. Health Res. 2012; 22 (1): 3-16. https://doi.org/10.1177/1049732311420448.

[11] Edwards I, Jones M, Higgs J, Trede F, Jensen G. What is collaborative reasoning? Advances in Physiotherapy. 2004; 6:70-83. https://doi.org/10.1080/14038190410018938.

[12] Gillespie M. Using the Situated Clinical Decision-Making framework to guide analysis of nurses' clinical decision-making. Nurse Education in Practice.2010; 10 (6): 333-40. https://doi.org/10.1016/j.nepr.2010.02.003.

[13] Hall MK. The Relationship between Perceived Barriers to Research Utilization Among Nonacute Care RNs and Telephonic Nursing Demographic Characteristics Using the BARRIERS Scale. Doctoral Dissertation. College of Management and Technology. Walden University. 2011.

[14] Haynes RB, Kastner M, Wilczynski NL, Team H. Developing optimal search strategies for detecting clinically sound and relevant causation studies in EMBASE. BMC Medical Informatics and Decision Making. 2005; 5(8):1-7.

[15] Higgs J, Jones MA, Loftus S, Christensen N. Clinical reasoning in the health professions. 3rd ed. Amsterdam: Elsevier Com., 2008, chapter 8; factors influencing clinical decision making. p:89-100.

[16] Hodgetts TJ, Kenward G, Vlackonikolis I, Payne S, Castle N, Crouch R, Shaikh L. Incidence, location and reasons for avoidable in-hospital cardiac arrest in a district general hospital. Resuscitation. 2002; $54 \quad$ (2): $115-23 . \quad$ https://doi.org/10.1016/S03009572(02)00098-9.

[17] Hoffman K, Elwin C. The relationship between critical thinking and confidence in decision-making. AJAN. 2004; 22 (1): 8-12.

[18] Hutchinson AM, Johnston L. Bridging the divide: a survey of nurses' opinions regarding barriers to, and facilitators of, research utilization in the practice setting. Blackwell Publishing Ltd, Journal of Clinical Nursing. 2004; 13 (3): 304-15 https://doi.org/10.1046/j.1365-2702.2003.00865.x.

[19] Jette DU, Bacon K, Batty C, Carlson M, Ferland A, Hemingway RD, Hill JC, Ogilvie L, Volk D. Evidence-based practice: beliefs, attitudes, knowledge, and behaviors of physical therapists. Phys Ther. 2003; 83(9):786-805.

[20] Kajermo KN, Nordstrom G, Krusebrant A, Bjorvell H. Barriers to and facilitators or research utilization, as perceived by a group of nurses in Sweden. Journal of Advanced Nursing. 1998; 27(4):79807. https://doi.org/10.1046/j.1365-2648.1998.00614.x.

[21] Kajermo KN, Unden M, Gardulf A, Eriksson LE, Orton ML, Arnetz BB. et al. Predictors of nurses_perceptions of barriers to research utilization. Journal of Nursing Management. 2008; 16(3):305-14. https://doi.org/10.1111/j.1365-2834.2007.00770.x.

[22] Kania-Lachance DM, Best PJM, McDonah MR, Ghosh AK. Evidence-based practice and the nurse practitioner. Nurse Pract. 2006; 31(10):46-54

[23] Lauri S, Salantera S, Chalmers K, Ekman S, Kim HS, Kappeli S. et al. An exploratory study of clinical decision-making in five countries. Journal of Nursing Scholarship. 2001; 33(1): 83-90. https://doi.org/10.1111/j.1547-5069.2001.00083.x.

[24] Lauri S, Salantera S. Developing an instrument to measure and describe clinical decision making in different nursing fields. Journal of Professional Nursing. 2002; 18: 93-100. https://doi.org/10.1053/jpnu.2002.32344.

[25] Ludin SM. Does good critical thinking equal effective decisionmaking amongcritical care nurses? A cross-sectional survey. Intensive and Critical Care Nursing. 2017; xxx () xxx-Xxx

[26] Majid Sh, Foo S, Luyt B, Zhang X, Theng Y, Chang YK, Mokhtar I A. Adopting evidence-based practice in clinical decision making: nurses' perceptions, knowledge, and barriers. Med Libr Assoc. 2011; 99(3): 229-36. https://doi.org/10.3163/1536-5050.99.3.010.

[27] Mantzoukas S. A review of evidence-based practice, nursing research and reflection: leveling the hierarchy. J ClinNurs. 2007; 17(2):214-23. https://doi.org/10.1111/j.1365-2702.2006.01912.x.

[28] McCaughan D, Thompson C, Cullum N, Sheldon TA, Thompson DR. Acute care nurses' perceptions of barriers to using research information in clinical decision-making. Blackwell Science Ltd, Journal of Advanced Nursing. 2002; 39(1), 46-60. https://doi.org/10.1046/j.1365-2648.2002.02241.x.

[29] Mehrdad N, Salsali M, Kazemnejad A. The spectrum of barriers to and facilitators of research utilization in Iranian nursing. Journal compilation. $\quad 2008 ; \quad 17(16)$ : $2194-2202$. https://doi.org/10.1111/j.1365-2702.2007.02040.x.

[30] Muntean WJ. Nursing Clinical Decision-Making: A Literature Review. 2012. Available at https://www.ncsbn.org/2388.htm

[31] Retsas A. Barriers to using research evidence in nursing practice. Journal of Advanced Nursing. 2000; 31(3): 599-606 https://doi.org/10.1046/i.1365-2648.2000.01315.x.

[32] Sarabia-Cobo CM, Sarabia-Cobo AB, Pérez V, Hermosilla C, Nuñez MJ, Lorena P. Barriers in implementing research among registered nurses working in the care of the elderly: a multicenter study in Spain. Applied Nursing Research. 2015; 28(4): 352-5. https://doi.org/10.1016/j.apnr.2015.03.003.

[33] Tan M, Akgun-Sahin Z, Kardas-Ozdemir F. Barriers of research utilization from the perspective of nurses in Eastern Turkey. Nurs $\begin{array}{lll}\text { Outlook. } & \text { 60(1):44-50 }\end{array}$ https://doi.org/10.1016/j.outlook.2011.07.002.

[34] Tanner C. Thinking like a nurse: a research-based model of clinical judgment in nursing. Journal of Nursing Education. 2006; 45 (6): 204-12.

[35] Thompson C, Cullum N, McCaughan D, Sheldon T, Raynor P. Nurses, information use, and clinical decision making--the real world potential for evidence-based decisions in nursing. Evid Based Nurs. 2004; 7: 68-72. https://doi.org/10.1136/ebn.7.3.68.

[36] Thompson C. Clinical experience as evidence in evidence-based practice. Journal of Advanced Nursing. 2003; 43(3), 230-237 235

[37] Woda A, Hansen J, Paquette M, Topp R. The impact of simulation sequencing on perceived clinical decision making. Nurse Education in Practice. 2017; 26(1):33-8. https://doi.org/10.1016/j.nepr.2017.06.008. 